\title{
A novel ternary CDMA code for TPSK modulation scheme
}

\begin{abstract}
In code division multiple access (CDMA), two or more chips are grouped together to form symbols and each symbol is transmitted during the symbol period. The phase shift keying (PSK) modulation techniques map the digital baseband data into two or more possible signals by varying the phase of a radio frequency (RF) carrier. The recently proposed PSK scheme called ternary PSK (TPSK) scheme can convey three possible symbols. In this paper, a novel ternary based CDMA sequence so-called large area synchronous even ternary (LAS-ET) sequence is introduced to increase spectrum efficiency in TPSK scheme. Its sequence duty ratio and cross-correlation are analyzed. The performance analysis of this sequence is compared with the large area synchronous (LAS) sequence in term of symbol error rate and chip error rate (CER) over various channel models. It is shown that TPSK scheme in LAS-ET sequence outperforms LAS sequence in terms of CER evaluation. At the same time, the spectrum efficiency is doubled when a pair of chips in LAS-ET sequence is mapped into one symbol.
\end{abstract}

Keyword: CDMA; CER; LAS; SER; TPSK 